\title{
Patterns of perceived similarity cannot be generalized from long to short exposure durations and vice versa
}

\author{
RAYMOND KLEIN \\ Dalhousie University, Halifax, Nova Scotia, Canada
}

\begin{abstract}
Subjects rated the perceived similarity between patterns and their 180-deg rotational variants. The stimuli, adapted from Halpern, Fishbein, and Warm (1979), were randomly generated dot patterns and line drawings and polygons generated from the dot patterns. Inspection time was varied in a between-subject design. As in the Halpern et al. (1979) study, it was found that the variables of form type (dots, lines, polygons) and axis of rotation $(x, y, z)$ had no effect on perceived similarity in the long exposure conditions. However, when inspection time was limited to $150 \mathrm{msec}$, both variables had a substantial impact. This finding undermines the frequent practice of selecting stimuli for use in reaction time or other experiments with limited inspection time on the basis of similarity ratings gathered under typical free inspection conditions.
\end{abstract}

How similar do two stimuli appear to an observer? What influence does a selected variable have on the pattern of perceived similarity within a set of stimuli? These questions are routinely posed by psychologists. In studies of visual perception, the answer is usually obtained by allowing an observer to freely examine a stimulus pair until he is ready to make his rating response. Information from a series of such judgments may be used to test a model of perception when that model makes predictions about perceived similarity. Similarity ratings may also be used in the stimulus selection phase of an experiment in order to equate similarity across stimulus sets, or vary it methodically. The purpose of this short report is to bring to the attention of psychologists who use similarity ratings our finding that stimulus duration has a profound effect on the pattern of perceived similarity. More specifically, at very short exposures $(150 \mathrm{msec})$, which are rarely used in ratings studies, perceived similarity is very sensitive to variables that have no effect at longer exposures. This finding undermines the common practice of selecting stimuli for use in reaction time and other experiments with brief exposure durations on the basis of similarity ratings gathered under free inspection conditions.

The stimulus patterns we used were taken from a study by Halpern, Fishbein, and Warm (1979) and are shown in Figure 1. These investigators were interested in the problem of representativeness in the perceived similarity of patterns. Their experiment compared the rated similarity of transformed stimuli

Thanks are extended to D. Halpern for providing the stimulus patterns used in this study. The author's complete address is: Department of Psychology, Dalhousie University, Halifax, Nova Scotia B3H 4J1, Canada. to standards (20 different transformations were tested) in three populations of forms (dots, lines, polygons). Dot patterns were generated by random selection of cells in an $8 \times 8$ matrix, while corresponding line and polygon patterns were created from the dot patterns by the application of specific rules (see Halpern et al., 1979, for a more complete description). Thus, a particular set of stimulus information is realized in three different graphic forms. Within each form type, subjects rated the similarity between the standard stimuli (shown in Figure 1) and each of 20 different transformations of these stimuli. The results revealed "a remarkable harmony in the distributions of ratings for the 20 transformations in each form population"' (Halpern et al., 1979, p. 25).

It was our intention to take advantage of this harmony by using the stimuli shown in Figure 1 in a chronometric study of mental size transformation. It has recently been suggested that subjects mentally transform the size of a presented pattern when they must determine whether two differently sized patterns have the same shape (Bundesen \& Larsen, 1975; Klein, Note 1). Examination of the literature suggests that the rate of mental size transformation may be influenced by configurational properties of the stimuli: the functions relating "same" RT to size ratio are steepest with dot patterns, shallowest with polygons, and intermediate with line drawings. Since many methodological differences between these studies preclude a firm conclusion, we sought to compare the three form types within a single experiment. Since "same" RT is affected by the similarity of the stimuli making up "different" trials, it is desirable to equate similarity between such stimuli across the three form types (dots, lines, and polygons). Halpern et al. (1979) demonstrated that the 


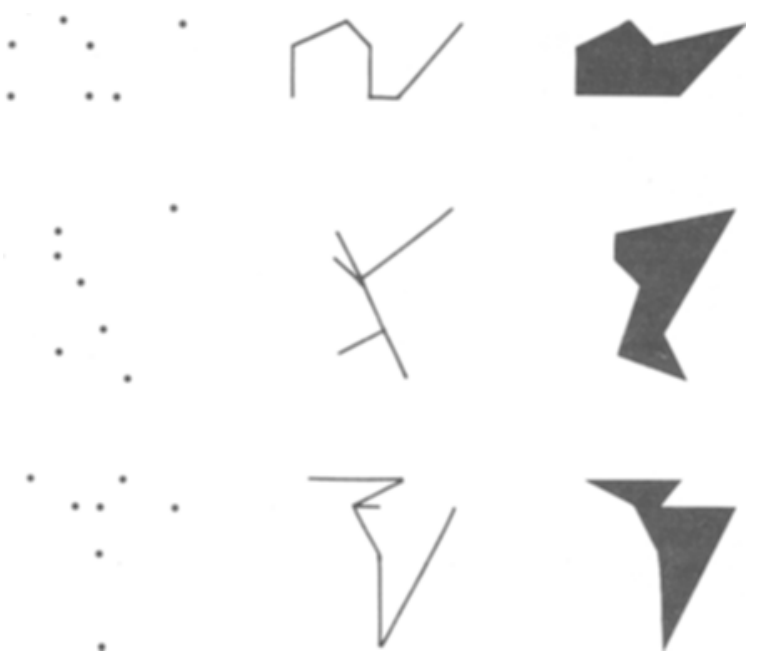

Figure 1. Nine standard stimuli used in the experiment. Each row shows the three patterns constructed from a given set of $x, y$ coordinates. Different form types (dots, lines, polygons) are shown in different columns.

perceived similarity between a standard pattern and each 180-deg rotation was the same whether the stimulus information was displayed as a dot pattern, line drawing, or polygon. Thus, by using their stimuli and the three 180-deg rotations about the major axes (their transformations 7, 11, and 12) for "different" trials, it would seem possible to equate "different" trial similarity across the three form types.

One salient difference between the Halpern et al. (1979) similarity study and our speeded performance task is the amount of time the stimuli are available to influence the subject's judgment or performance. In the Halpern et al. (1979) study, subjects were free to examine the stimuli for up to $8 \mathrm{sec}$, while in our speeded matching task response latencies ranged from about 800 to $1,800 \mathrm{msec}$, and the time during which perceived similarity might influence performance was necessarily shorter than this. Is it valid to assume that patterns of perceived similarity obtained under the "leisurely" inspection conditions of a psychophysical experiment will be maintained when inspection time is very limited? If not, then the applicability of similarity rating data obtained under typical (long exposure) conditions to studies of performance under speed stress will be seriously questioned. To answer this question, we obtained similarity ratings between the nine standard stimuli shown in Figure 1 and each of three 180-deg rotations under three exposure duration conditions: (1) up to $8 \mathrm{sec}$, or until a response, (2) $1,500 \mathrm{msec}$, and (3) $150 \mathrm{msec}$.

\section{METHOD}

\section{Subjects}

Twelve male and 12 female students at Dalhousie University served as subjects. They either volunteered or participated for course credit.

\section{Stimuli and Apparatus}

Each of the nine stimuli shown in Figure 1 was paired with each of three 180-deg rotations (about the $x-, y-$, and $z$-axes) to create 27 stimulus pairs. Three of these are shown in Figure 2, each panel of which illustrates a different pattern, form type, and type of rotation. The 27 stimuli were randomized and presented in the same order for each subject.

The stimuli were rear-projected onto a $50 \times 40 \mathrm{~cm}$ gray translucent screen by a Kodak $860 \mathrm{H}$ Carousel projector. The subjects sat approximately $120 \mathrm{~cm}$ from the screen. At this viewing distance, a single pattern subtended about $4 \mathrm{deg}$, and its near edge was about $3 \mathrm{deg}$ from fixation. The two patterns appeared side by side centered about a small dot on the screen. A tachistoscopic shutter (Gerbrands G1166) was used to control exposure duration, and a response key was used by the subject to initiate each trial and to terminate exposure in the long-duration condition. The projector, shutter, and response key were connected to a PDP 11-10 computer, which was used to control the sequence of events.

\section{Task and Procedure}

The subject's task was to rate the similarity between the two members of a stimulus pair. This was done after each stimulus presentation by making a mark on a line labeled from 0 to 100 in steps of 5 . The subjects were instructed that 0 represented minimum similarity and 100 represented maximum similarity.

Upon fixating a small dot on the center of the screen, the subject initiated each trial by pressing the response button. Two seconds later, the stimulus pair was presented for $150 \mathrm{msec}$, $1,500 \mathrm{msec}$, or up to $8 \mathrm{sec}$, depending upon the exposure condition. In the long $(8 \mathrm{sec})$ exposure condition, the subject was instructed to terminate the exposure when he was ready to make his rating response.

\section{Design}

The three different form types (dots, lines, and polygons) and three axes of rotation (about $x-, y-$, and $z$-axes) were within-subject variables. Exposure duration and sex were between-subjects variables. Four male and four female subjects were assigned randomly to each exposure condition.

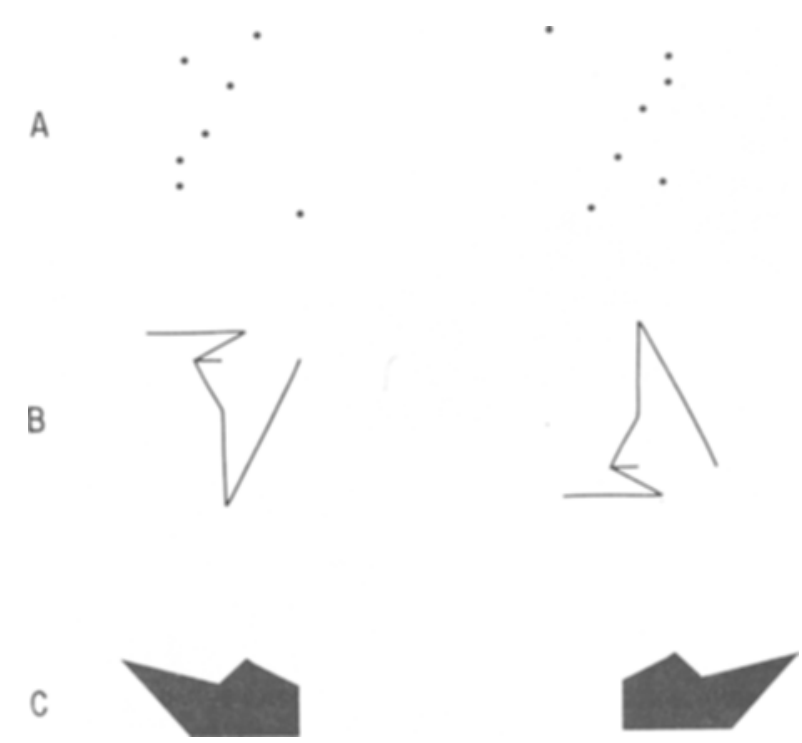

Figure 2. Examples of stimulus pairs used in the experiment. (a) z-axis rotation, dots; (b) $x$-axis rotation, lines; (c) $y$-axis rotation, polygon. 


\section{RESULTS AND DISCUSSION}

The ratings for the three different patterns were averaged for each subject and for each combination of form type and axis of rotation. These average scores were subjected to a $3 \times 2 \times 3 \times 3$ mixed ANOVA, with exposure duration and sex the between-subjects factors and form type and axis of rotation the withinsubject factors.

The main effects of exposure duration $[\mathrm{F}(2,18)=$ $22.86, p<.01]$ and form type $[F(2,36)=5.29, p<.05]$ were significant. The main effect of type of rotation was only marginally significant $[F(2,36)=2.56, p<.1]$. The two-way interactions between exposure duration and form population $[F(4,26)=6.24, p<.01]$ and exposure duration and type of rotation $[F(4,36)=$ $5.25, \mathrm{p}<.01]$ were significant. No other main effects or interactions approached significance.

All the main findings of interest can be seen in Figure 3. As one might expect, perceived similarity increases with exposure time. More important is the finding that the two variables of interest, form type (3a) and axes of rotation (3b), which have no effect on perceived similarity at the long exposure durations, have a substantial impact when the stimuli are presented for only $150 \mathrm{msec}$. One can summarize this impact by saying that the depressing effect of short exposures upon perceived similarity is greatest for dot patterns and $\mathrm{x}$-axis rotations and least for polygons and $y$-axes rotations.

The methodological significance of this finding is straightforward: the pattern of perceived similarity that is observed under typical long-exposure conditions cannot be generalized to situations in which processing time is very limited. The theoretical significance of the emergence of similarity effects at short exposures will depend upon one's interpretation of the underlying cause of the effect.

In considering such an interpretation, it is interesting to note that the similarity differences produced by different form types at the shortest exposure duration are similar to mental size transformation rate differences that have been observed with the same material. In studies of mental size transformation, for example, the functions relating reaction time to size ratio are steepest for dot patterns, shallowest for polygons, and intermediate for line drawings (see Table 1). It seems reasonable to propose that perceived similarity between a pattern and its transform will depend upon the ease and/or likelihood of performance of the reverse or rectifying "mental" transformation. By allowing plenty of time for the performance of the mental transformation, the typical "leisurely" psychophysical procedure for obtaining similarity ratings would restrict data to an asymptotic region. That is, a ceiling effect would obscure differences in perceived similarity between patterns differing by a transformation. Very
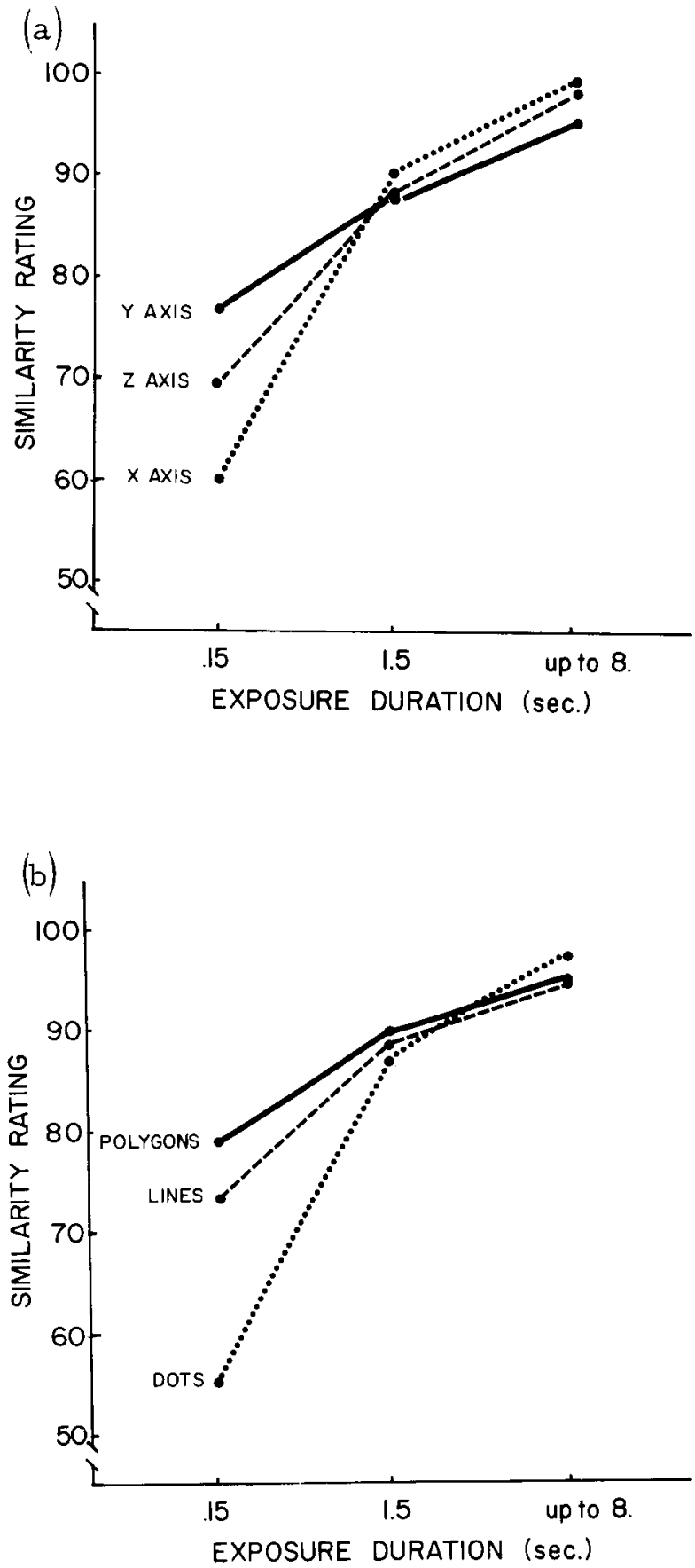

Figure 3. Similarity ratings as a function of exposure duration and (a) form type and (b) axis of rotation.

short exposures do not allow sufficient time for the mental transformation and therefore tap a similarity structure that is sensitive to transformational difficulty and more appropriate for use in studies or models dealing with time-based mental operations.

Of course, other lines of argument might be followed. For example, many models of perception propose that qualitatively different information about a 
Table 1

Size Transformation Rates for Different Form Types ("Same” Data Only)

\begin{tabular}{|c|c|c|c|}
\hline Source & Type of "Different" Trials & Complexity & Slope (in Milliseconds) \\
\hline \multicolumn{4}{|c|}{ Dots } \\
\hline Klein (1979) & Unrelated & $\begin{array}{r}5 \\
10\end{array}$ & $\begin{array}{l}150 \\
200\end{array}$ \\
\hline Starratt (1980): Experiment 1 & $\begin{array}{l}\text { Unrelated } \\
\text { Rotated }\end{array}$ & $\begin{array}{l}7 \\
7\end{array}$ & $\begin{array}{l}190 \\
150\end{array}$ \\
\hline Starratt (1980): Experiment 3 & $\begin{array}{l}\text { Unrelated } \\
\text { Unrelated } \\
\text { Unrelated }\end{array}$ & $\begin{array}{r}5 \\
10 \\
15\end{array}$ & $\begin{array}{l}150 \\
210 \\
270\end{array}$ \\
\hline Starratt (1980): Experiment 5 & Rotated & 7 & 224 \\
\hline \multicolumn{4}{|c|}{ Lines } \\
\hline Bundeson \& Larsen (1975): Experiment 1 & Unrelated & 6 & 75 \\
\hline Bundeson \& Larsen (1975): Experiment 3 & Unrelated & 6 & 50 \\
\hline Besner \& Coltheart (1976) & Unrelated & 6 & 90 \\
\hline Starratt (1980): Experiment 5 & Rotated & 7 & 77 \\
\hline \multicolumn{4}{|c|}{ Polygons } \\
\hline Bundeson \& Larsen (1975): Experiment 2 & Rotated & 9 & 30 \\
\hline Starratt $(1980)$ & Rotated & 7 & 40 \\
\hline
\end{tabular}

Note-Complexity is defined as the number of $x, y$ coordinate pairs used to generate the pattern. In some cases, slope was estimated from figures.

stimulus is available at different times after its presentation. Although exactly how these models would account for our results would need to be worked out, their basic tenet is certainly consistent with our findings. It may also be possible to develop an account which places importance on visual inspection of the to-be-judged stimuli by saccadic eye movements. In the 150-msec exposure condition, there is insufficient time for any saccades - a fact that could be responsible for the emergence of similarity effects.

In summary, our results in the long-exposureduration conditions clearly confirm those of Halpern et al. (1979) in showing no effect of form type or axis of rotation on the similarity between rotated patterns. However, when we used 150-msec exposure durations, both type of rotation and form type had large effects on perceived similarity. While, on the basis of our research, a theoretical interpretation of this finding can only be suggested, the methodological implication is clear: patterns of perceived similar- ity cannot be generalized from short to long exposure conditions, and vice versa.

\section{REFERENCE NOTE}

1. Klein, R. Explorations of mental size scaling. Paper presented at the American Psychological Association, New York, August 1979.

\section{REFERENCES}

Besner, D., \& Coltheart, M. Mental size scaling examined. Memory \& Cognition, 1976, 4, 525-531.

Bundesen, C., \& Larsen, A. Visual transformation of size. Journal of Experimental Psychology: Human Perception \& Performance, 1975, 1, 214-220.

Halpern, D., Fishbein, H., \& W ARM, J. Similarity judgments of patterns and maps. Bulletin of the Psychonomic Society, $1979,13,23-26$.

Starratt, G. Cognitive processes involved in size transformation. Master's thesis, Dalhousie University, 1980.

(Manuscript received January 13, 1982; revision accepted for publication February 26, 1982.) 